\section{RE-CAPTURING A LOST POPULATION - THE BENEFITS OF A NURSE-DELIVERED HCV COMMUNITY CIRRHOSIS CLINIC}

Maryam Noeman, Sehar Farooq, Lourdes Cumlat, Rajan N Patel ${ }^{*}$, William Blad, Indrajit Ghosh, Deepak Suri. The Whittington Hospital NHS Trust, London, UK

\subsection{6/gutjnl-2020-bsgcampus.72}

Introduction The current landscape of service provision for patients with liver disease does not match that of disease burden. A North London-based drug and alcohol dependency unit ('Better Lives') provides blood borne virus (BBV) screening and opioid substitution therapy (OST) as well as Hepatitis $\mathrm{C}$ Virus (HCV) treatment. These patients are often complex with marginalised social needs, have chaotic lifestyles and don't engage with conventional care models. We aimed to describe the demographics of a cohort of patients seen in a novel community outreach clinic (OC).

Methods Approximately 1000 patients per year receive OST from 'Better Lives' via a multidisciplinary clinic with doctors, nurses and recovery workers. $117(11.7 \%)$ screened positive for chronic HCV. 22 (18.8\% of chronic HCV patients, 2.2\% of those receiving OST) with advanced liver fibrosis or cirrhosis (F3/F4) were referred to OC between November 2018 and September 2019. We used electronic hospital records to assess 'Did Not Attend' (DNA) rates to outpatient appointments (OPA) and emergency department (ED) attendances prior to review in $\mathrm{OC}$ and completion of key investigations afterward.

Results 22 patients referred to OC in the study period (median age 52 (IQR 46.3 - 57), 81.8\% male, 40.9\% white). 14 patients $(63.6 \%)$ attended. Chronic HCV primary aetiology in $12(54.5 \%), 11(50 \%)$ self-reported ongoing alcohol intake and $4(18 \%)$ were people who inject drugs (PWID). Median liver stiffness $19 \mathrm{kPa}$ (IQR 12.95 - 36.1). 12 (54.5\%) did not have registered GPs. 15 (68.2\%) DNA hospital appointments prior to OC clinic.

Conclusion This is the first UK-based nurse-delivered community liver cirrhosis outreach clinic aimed to improve treatment and supportive medical care and offer a link to hospital cirrhosis services. We re-engaged two-thirds of complex marginalised patients with advanced fibrosis via OC that would otherwise have been lost to follow up. Patients attending OC had an average of 2 prior DNA to hospital OPA and 6 ED attendances. $35 \%$ and almost $50 \%$ of patients had their surveillance US abdomen and gastroscopies, respectively, following

Abstraact 072 Table 1 Comparison between patients attending outreach clinic and not attending

\begin{tabular}{|c|c|c|c|}
\hline & $\begin{array}{l}\text { All F3/F4 patients } \\
\text { referred } \\
(n=22)\end{array}$ & $\begin{array}{l}\text { Patients attending } \\
O C(n=14)\end{array}$ & $\begin{array}{l}\text { Patients not } \\
\text { attending OC } \\
(\mathrm{n}=8)\end{array}$ \\
\hline HCV Aetiology, n (\%) & $12(54.5)$ & $9(64.2)$ & $3(37.5)$ \\
\hline $\begin{array}{l}\text { Prior DNA OPAs, } \\
\text { mean (SD) }\end{array}$ & $2.14(2.14)$ & $2.07(1.64)$ & $2.25(2.96)$ \\
\hline $\begin{array}{l}\text { Prior ED attendances, } \\
\text { mean (SD) }\end{array}$ & $5.05(6.94)$ & $6(7.2)$ & $3.38(6.57)$ \\
\hline $\begin{array}{l}\text { US Abdomen after } \\
\text { OC, } n,(\%)\end{array}$ & $7(31.8)$ & $6(42.86)$ & $1(12.5)$ \\
\hline $\begin{array}{l}\text { Gastroscopy after } \\
\text { OC, n (\%) }\end{array}$ & $6(27.3)$ & $5(35.71)$ & $1(12.5)$ \\
\hline
\end{tabular}

attendance to OC compared with only $12 \%$ who did not attend. Further work to investigate if a community based liver outreach clinic can improve the trajectory of marginalised patients is required.

\section{3 INTRODUCING A CLINICAL NURSE ENDOSCOPIST LED BARRETT'S SURVEILLANCE PROGRAMME IN A TERTIARY REFERRAL CENTRE}

Sara Elizabeth May Brogden*, Rehan Haidry. Uclh, London, UK

\subsection{6/gutjnl-2020-bsgcampus.73}

Introduction Endoscopic eradication therapy (EET) is established as the first line therapy for patients with Barrett's oesophagus (BE) related dysplasia to avoid progression to invasive cancer. Success rates for disease reversal are high but there is an appreciable risk of recurrence. Periodic endoscopic surveillance is mandated to detect disease recurrence early so further curative EET is possible.

After successful EE, endoscopic surveillance is mandated by all international societies to detect recurrence. This can however be time and resource intensive and can impact on already busy therapeutic endoscopy services. Therefore exploring how to divert these patients to other bespoke sessions is needed, thereby allowing the specialist consultant operators to focus on delivering therapeutic endoscopy to maximise resources and enhance patient care.

Aim and Methods Consecutive patients with BE at a London specialist tertiary hospital with over 10 years' experience in delivering EET were analysed. All patients had achieved successful EET for BE neoplasia and were entered in a post treatment surveillance programme.

Gastroscopies were performed on a dedicated Clinical Nurse Endoscopist (CNE) BE surveillance sessions.

The CNEs had at least 5 years expertise in BE surveillance and specialist ongoing training by the clinical lead. Three dedicated surveillance sessions were performed weekly with an average of 4-5 patients per endoscopy session.

Data was collected over a three year period 2016- 2019, a total of 456 patients were analysed.

All procedures were carried out using white-light, NBI endoscopy and acetic acid chromoscopy where indicated and biopsies performed as per post EET surveillance protocols and reviewed by dedicated pathologist with an interest in BE.

All patients with relevant histopathology changes or concerning features were discussed in dedicated multidisciplinary meetings.

Results 456 patients were analysed (x males, $\mathrm{x}$ female, median age $\mathrm{xxx}$ )

Over a 36 month period the dedicated CNE programme was able to detect low grade dysplasia (LGD) in 26 patients, high grade dysplasia (HGD )14 patients, Indefinite for dysplasia (ID) 34 patients and inter mucosal carcinoma (IMC) in 2 patients.

Overall dysplasia recurrence was $15.7 \%$ in this cohort of patients and all were offered retreatment with EET

The data also shows that out of 456 patients undergoing a gastroscopy only 1 needed repeating by a consultant due to abandoning the procedure.

Conclusion We retrospectively analysed consecutive cases with BE following successive EET in a dedicated and specialist CNE led surveillance service in a tertiary referral centre. 